\title{
Computing on the Desktop: From Batch to Online in Two Large Danish Service Bureaus
}

\author{
Anker Helms Jørgensen \\ IT University of Copenhagen \\ Rued Langaardsvej 7, DK-2300 Copenhagen S, Denmark \\ ankereitu.dk
}

\begin{abstract}
This paper addresses the change from batch mode to online mode in offices and workplaces in the 1970s and 1980s. Particular focus is on the role of IBM and the 3270 display terminal. The change had profound implications for users and system developers. The paper is based on oral history interviews with former employees of the two large Danish service bureaus, Datacentralen and Kommunedata, and addresses a number of themes: driving forces, technology, education and training, skills and knowledge, and systems development practice.
\end{abstract}

Keywords: Batch, display terminal, IBM 3270, online.

\section{Introduction}

The term "computing on the desktop" is today associated with the personal computer. Indeed, the personal computer resides on many desktops and has been doing so for many years. However, the desktop was invaded by computing facilities in the decades before the personal computer. The display terminal made its way to offices and workshops in the 1970s and 1980s, facilitated by emerging timesharing capabilities, increasingly powerful computers, the growing need for fast access to information, and more user-friendly types of dialog.

This introduction implied a considerable change for users and system developers. The former batch processing mode involved cumbersome and slow punch card technology, huge piles of print, and turn-around times counted in hours, while in online mode users could access information quickly. This development took place over several decades. Although online technology matured in the 1960s, the change from batch to online did not take place in most organizations until the 1970s and 1980s. As an example, online access only became available in the Danish Civil Registration System (CPR-register) in 1981, although the system had already been established in 1968 [1].

In these decades the state-of-art in commercial computing has been called "IBM and the seven small dwarfs" [2, p. 129], referring to the dominant role IBM had with its market share of 60 to 70 percent. The foundation was laid when IBM released the groundbreaking, general purpose IBM 360 computer in 1964. It was designed to 
handle batch and online alike, but performed poorly in the latter [3, p. 155], which later versions of the 360 and in particular the 370 remedied. IBM also played a significant role in the proliferation of online computing, with the 3270 display terminal family, released in 1971 [4].

As to significance of the display terminal at large, I will make a comparison with other significant technologies. Mike Mahoney [5] compared IBM to the canonical Ford T: "Much as Henry Ford taught the nation to use an automobile (by way of Ford T), IBM and its competitors taught the nation's businesses (and government) how to use the computer." Accordingly, the basic thesis in this paper is that IBM and its competitors taught employees in the nation's businesses to use the computer in online mode, largely with the IBM 3270 and compatible display terminals, backed by the dominance of the IBM 360 and 370 computers. The paper is an initial attempt to create grounds for substantiating this basic thesis. A twofold approach is used: literature study and oral history - later to be supplemented by archive studies.

\section{Batch Mode and Online Mode}

Batch is a mode of operation with a computer, where programs or systems ("jobs") are executed without human intervention. This mode is well suited to high-volume, repetitive processing. Originally, mainframe computers only employed this mode. The typical input media were magnetic tape and punch cards, prepared by punch card operators, while the typical output medium was punch cards and print, often in massive amounts. The turn-around time was counted in hours. Batch designated processing of a large amount of data usually occurred at regular intervals: daily, weekly, or monthly. Working with the information involved huge piles of printed material, so-called Leporello lists. As batch jobs were run infrequently, the administrative routines supported were not necessarily based on up-to-date data. Although the online mode is prevalent today, batch is still alive and well. It is employed for background processing such as database updating, report generation, and high volume printing.

Online is a mode of operation where the human user interacts directly with the system, using either a screen or a paper terminal. ${ }^{1}$ Processing took place one-at-a-time in real time - as opposed to batches - with data stored on disks or diskettes with (almost) instant access time. Working online implied that information was up-to-date and answers to queries were provided immediately. Response time was counted in seconds.

As to the novelty of the online mode, William D. Orr stated "conversational interaction with computers is a major new phenomenon in its own right, not simply another 'application' of computers" [6]. A way of characterizing the differences is suggested by Long and colleagues [7, p. 39]: "In batch systems, the end-user's relationship with the computer is indirect. Specialists write the programs, enter the data and operate the machine ... the role of the user is restricted to specifying the task

${ }^{1}$ Although the Teletype (TTY) played a significant role as terminal in the 1970s and 1980s, I have decided to focus solely on the display terminal as the visual appearance has survived into today's interactive systems. 
in sufficient detail for the computer specialists to do their work." This statement points to the heart of the distinction between batch and online, namely the directness and connectedness in the dialogue.

While the turn-around time in batch mode was several hours, the interaction in online mode took place "instantly." But "instantly" was not necessarily instantly. Response time was often unbearably slow and highly variable, hence response time became crucial and the subject of research [8]. This variability made Nobel Laureate Herbert Simon suggest that the system should deliberately delay the response to ten minutes, if the system could not respond within a short timeframe [9].

Throughout the 1960s, the interest in batch mode and online mode was significant. Harold Sackman conducted a series of studies on programmer productivity in the two modes, finding that online programming was in general quicker. However, the most important finding was the large differences between individual programmers: up to a factor of ten [10].

The advent of online mode incurred a flurry of papers and reports addressing usersystem dialog design. A landmark book appeared in 1973: James Martin's Design of Man-Computer Dialogues [11]. The book listed numerous dialog techniques and included a section on psychological considerations. Papers and reports focusing on dialog design guidelines appeared, such as the one by Engel and Granda [12]. These guidelines were aggregated in huge reports in the 1980s, culminating in 1986 with Guidelines for Designing User Interface Software by Smith and Mosier [13]. This report contained no less than 944 guidelines.

The Sackman studies and the guideline endeavors were examples of forerunners of a new academic field: Human-Computer Interaction (HCI). The focus of HCI is the interaction between a human user and the computer system, connected through a display terminal. The seeds emerged in the 1970s, but the field gained momentum in the 1980s. Oddly enough, in batch mode there was also significant interaction between the user and the computer, albeit much more indirect and asynchronous. Nevertheless, apart from a very limited number of studies, this interaction was not given much attention by researchers.

\section{The 3270 Display Terminal and Protocol}

The 3270 terminal family was manufactured from 1971 to 1977 , ending with the 3278 and 3279 high resolution display terminals with colors and graphics [4]. As the first 3270 display terminals were monochrome (green), they were called "green screens" and referred to as "dumb" terminals. The latter is true from today's perspective, but the 3270 terminal was by then not "dumb," since it came with an efficient protocol based on data streams - not unlike today's HTTP-protocol used in World Wide Web - and an innovative small memory facilitating local operations, such as syntax checking. This solution served to off-load the central computer and the communication lines. The 3270 protocol continued to live - a witness to technological momentum. Even today, many applications rooted in the 1970s and 1980s are using the 3270 protocol emulated on PCs. As part of their approach towards online computer use, IBM presented Systems Network Architecture (SNA) in 1974, which significantly advanced the state of the art in teleprocessing software systems. 
As IBM's blueprint for future teleprocessing products, SNA provided a unified design for IBM's data communications products [14, p. 399]. It is noteworthy that the 3270 display terminal family, the 3270 protocol, and SNA all adhered to the principle behind the successful IBM 360 computer: uniformity and generality.

\section{Two Large Danish Service Bureaus}

Datacentralen (now CSC) and Kommunedata were cutting edge service Bureaus in the Danish data processing business, established in 1959 and 1972 respectively. Together they covered a considerable share of the data processing needs of public authorities, Datacentralen aiming primarily at Government institutions and Kommunedata at regional and local authorities [15-17]. The term "service bureau" calls for a comment as Kommunedata and Datacentralen both undertook substantial software development in addition to operations and service.

\section{An Exploratory Pilot Study}

In order to probe the general attitude to the 3270 era, I conducted an informal survey among five experienced IT-colleagues, eliciting their free association responses to the question: "When I say 3270, what do you say?" The following comprises the essence of their answers:

- It was a dumb terminal and at the same time the first intelligent terminal

- It set the standard for a long time and had a fantastic lifetime as protocol

- It employed full screen mode, but validation of fields was difficult

o The navigation was difficult and a nuisance for users for many years

These answers supported the planning of the interviews presented in the next section.

\section{Oral History Interviews}

Three staff members or former employees at Kommunedata were interviewed on June 1, 2010. Employed in 1957, 1967, and 1974 respectively, they had occupied various positions: punch card operator, plug-board programming, programming, management, project management, servicing clients, and SAP customization. The interview, which took 1.5 hours, was recorded and shortly after transcribed.

One ex-staff member of Dacatentralen was interviewed on June 16, 2010. He had been employed at Datacentralen since 1980 and had worked with programming, testing, standards development, teaching, and technical writing. This interview also took 1.5 hours. The main interview themes were: driving forces, technology, education/training, skills and knowledge, and systems development practice. The interview focused on batch and online, but a lot of other aspects were covered, some of which are included below, in the interest of context.

Both companies primarily employed IBM and IBM-compatible hardware, soft-ware, and display terminals (Stansaab Alfaskop, fully 3270-compatible). 
Kommune-data used assembly language at an early stage. PL/I, along with COBOL, entered the scene with the online operations and SAP later became the overall development framework.

The transition from batch to online came in many disguises. One of the first steps in Kommunedata was installing a display terminal in a municipality and providing the option of requesting the "instant execution" of jobs. Therefore, instead of turn-around times of a day or so and waiting for the mail to deliver the paper output, the users in the municipality could see the result of the job execution via their terminal, immediately after the "instant execution." This mode resembled a hybrid of batch and online operations - of which many existed [18]. Similarly, at Datacentralen, a hybrid form of queuing online requests and processing them in small, fast batch jobs was employed. In addition, at Datacentralen, a fast punch card terminal was installed, which reduced turn-around time considerably. On a good day, one could submit one's stack of cards, walk to the print desk and watch the print appear.

Interestingly, a major influence on online operations was bills passed in the Danish parliament. In the 1960s, two major reforms were implemented: the Danish Civil Registration System (CPR-register) in 1968, upon which the Pay-as-You-Earn Taxation scheme of 1970 was based. These batch systems and the accompanying opportunities helped in bolstering the need for local municipalities to access centrally stored data online, as every citizen in Denmark was given a unique identification number.

Other incentives included the internal competition among departments in Kommunedata to provide the municipalities with the most advanced systems, and the inspiration resulting from the regular trips to IBM sites in the US, where Kommunedata staff, at the invitation of their primary supplier IBM, was presented with the newest developments. In addition, IBM handled almost all the education of Kommunedata personnel such as project management courses at Lidingö in Sweden.

In spite of the interest and pressure from many municipalities, there was also resistance, not least in small municipalities. During a presentation of the features of online access for the board of a municipality in Southern Jutland, a member of the board insisted: "Three second response time ... it can't be done ... it is a lie!" He was heading the IT Department of a local enterprise that had not yet discovered the virtues of online access. At the same event, the mayor questioned the need for such rapid access in the social sector. A bright member of the Kommunedata team replied that everybody could become a social case, fast handling time was in the interest of the person in question - hinting at the mayor himself.

With regard to the system developers and programmers, one of the first consequences of the introduction of display terminals in Kommunedata was that they had to punch their programs themselves instead of punch card operators carrying out the task. However, even after the introduction of the terminals, they asked the best punch card operators to punch their programs, as these operators were very skilled in detecting syntactic errors in the code. The most significant change for the programmers was turn-around time. With batch development, they had one - or sometimes two or even three - shots per day. This meant that a lot of time was spent on hand-testing the programs, but with online access this discipline soon vanished. Nevertheless, the conditions in the early days were far from those of today, since there could be as many as twenty-five developers sharing one display terminal. 
Regarding user interface design, the Kommunedata employees admitted that some of the 3270 applications were not that self-evident and user friendly. The user interface design skill and experience gradually evolved during the transition to online operations, without any external sources such as literature, conferences, and consultants seemingly having played a role. The attitude was that a system should be a natural part of the users' workday, requiring user involvement. As an example, the sequence of the fields on the screen, which is of great importance to users' everyday activities, was mentioned. No particular activities, such as courses, standards, handbooks or the accumulation of systematic experience, seem to have been established early on, but user interface standards did emerge later. When the focus on user interfaces was peaking in the 1990s, special dialog designers were used in Kommunedata. In user interface design, one of the most fundamental principles is consistency, which is manifested in organizations as standards. In Datacentralen, agreement on a user interface standard was a bumpy process that lasted three years. Regarding the organizational push for user friendliness, the creation of the Usability Test Center in the 1990s, at Kommunedata in Copenhagen, resulted in the publishing of guidelines. This was accompanied by fairly strict requirements regarding usability testing, which were later eased. With the current SAP foundation, this has changed; in the words of a SAP customizer: "We can fiddle a bit here and there, do things a bit smarter, but the options are really limited."

In the early days at Kommunedata, there was close contact between the developers and the customers and users in the municipalities. These would even call the Kommunedata staff and abuse them, if they had made errors. The developers took pride in what they did - later, a certain degree of alienation crept in. Gradually, the collaboration became more organized and formalized, not least when the Association of Danish Municipalities (KL) was established in 1970. Steering committees were established that worked out the specs between users and developers jointly, primarily focusing on functionality. The developers wrote the specs and the users were not really involved in the design of the user interface.

As mentioned before, the 3270 display family and protocol were introduced in 1972. The "green screens" are long gone, but the protocol is not. A good number of 3270-based applications are still operational at Kommunedata, simulated on PCs. In many of these older applications, the central components are from the 1970s or 1980s. Some PL/I -based applications also still exist, as well as some applications where the kernel is written in assembler. Therefore, as history evolves, software persists, but hardware disappears. This also happens to people; assembly programmers are becoming rare - as are PL/I programmers.

At times, the introduction of display terminals evoked odd situations. In planning a new building, some employees strongly argued that space for one display terminal for every three developers had to be allocated, a hard to win argument, but they succeeded.

At Datacentralen, two local software systems that support online operations were developed in 1973 (DDBS and TDSS), to facilitate telecommunication-based access to databases. The reason was the lack of suitable software from IBM. In a later heroic effort, the developers of these systems "shoehorned" these systems into the general IBM online Information Management System (IMS). 
With regard to the interview mentioned previously, a question was also included concerning the significance of the transition from batch to online in the broader perspective of important innovations over the last sixty years, such as miniaturization, client/server architecture, the personal computer, the graphical interface, and the Internet. The Kommunedata employees agreed that the significant change was the personal computer, not the display terminal. However, being in the middle of it in the 1970s and 1980s, the display terminal was a big thing. The Datacentralen interviewee responded to this question in terms of database access (DB2) - an accompanying and necessary feature of online mode. However, when I repeated the question the interviewee stated that the display terminal was a revelation and thereby significant.

\section{Discussion and Conclusions}

Due to the limited number of interviewees, the paper does not warrant strong conclusions, but a few points are worth mentioning. First, the transition involved a number of batch/online hybrid forms - of which this study has undoubtedly only shed light on a few; see [18] for another survey. This calls for a comment on the classical question of evolution or revolution in technology and computer history. I think is safe to say that the batch/online transition took place in a number of different stages and that the display terminal has paved the evolutionary path for the personal computer with its much more elaborated capabilities. Perhaps it may even be more meaningful to think of the "batch to online" issue as "batch and online"? It seems that the new craft of online user interface design was not generally informed by existing knowledge sources such as literature, courses, and consultants. User interface design was approached locally, perhaps another manifestation of the "not invented here" syndrome not unknown in the IT realm. Throughout the interviews, it was often difficult to maintain focus on the core issue from batch to online, such as user interface design in online mode and the accumulation of experience from this novel aspect of development. It seems that this central theme has not played an important role in the interviewees' professional lives. Nevertheless, it seems that the introduction of the display terminal and online operations has had a significant impact on users and developers alike and that IBM played a significant role in this transition.

Acknowledgments. I thank Søren Lauesen, Rolf Molich, Peter Carstensen, and Jan Clausen for comments on an earlier version of this paper.

\section{References}

1. Nielsen, H.: CPR - Danmarks Folkeregister. CPR-Kontoret (April 1991), http: //www.cpr.dk/cpr_artikler/Files/Fil1/4392.pdf (accessed on June 10, 2010)

2. Campbell-Kelly, M., Aspray, W.: Computer: A History of the Information Machine. Westview Press, Boulder (1996)

3. Ceruzzi, P.E.: A History of Modern Computing. MIT Press, Cambridge (1998) 
4. IBM 3270 Information Display System Component Description. Report IBM GA 27 2749-1, 1972. IBM Systems Development Division, Product Publications, Kingston, NY 12401

5. Mahoney, M.S.: The History of Computing in the History of Technology. Annals of the History of Computing 10, 113-125 (1988)

6. Orr, W.D. (ed.): Conversational computers. Wiley, Chichester (1968)

7. Long, J., Hammond, N.V., Barnard, P., Morton, J., Clark, I.A.: Introducing the interactive computer at work: The users' views. Behaviour and Information Technology 2, 39-106 (1983)

8. Miller, R.B.: Response time in man-computer coversational transactions. In: AFIPS Fall Joint Computer Conference, vol. 33, part 1, pp. 267-277 (1968)

9. Simon, H.A.: Reflections on time sharing from a user's point of view. Computer Science Research Review, 43-51 (1966)

10. Sackman, H.: Man-computer problem solving. Auerbach, New York (1970)

11. Martin, J.: Design of Man-Computer Dialogues. Prentice-Hall, Englewood Cliffs (1973)

12. Engel, S.E., Granda, R.E.: Guidelines for Man/Display Interfaces. Report TR 00.2720. Poughkeepsie, NY, IBM (1975)

13. Smith, S.L., Mosier, J.N.: Guidelines for designing user interface software. The MITRE Corporation, Bedford Mass, USA, rapport ESD-TR-86-278 (August 1986)

14. Jarema, D.R., Sussenguth, E.H.: IBM data communications: A quarter of evolution and progress. IBM Journal of Research and Development 25(5), 391-404 (1981)

15. I/S Datacentralen af 1959 igennem 25 år. Datacentralen, Copenhagen (1984)

16. Falk, R.: Fra hulkort til EDB: Jyske Kommuners Hulkortcentral. Aalborg Stadsarkiv and KMD (2006)

17. Laursen, A.: Historien om en central. Kommunedata, Aalborg (1991)

18. Jørgensen, A.H.: Exploring the Transition From Batch to Online: Datamation as Source of Evidence. In: Hertzum, M., Hansen, M. (eds.) Proc. DHRS 2010: 10th Danish HumanComputer Interaction Research Symposium, Roskilde, pp. 27-30. (November 2010), http://magenta.ruc.dk/dat_en/research/reports/132/ (accessed November 21, 2010) 